\title{
Acute kidney injury in severely injured patients admitted to the intensive care unit
}

\author{
Alberto F. García 1,2, Ramiro Manzano-Nunez ${ }^{3,4^{*}}$, Juan G. Bayona ${ }^{1,2}$, Maria P. Naranjo 1,2, Dary Neicce Villa1,2, \\ Manuel Moreno ${ }^{5}$, Sebastian Ossa ${ }^{5}$, Juan M. Martinez ${ }^{5}$, Nathalia Martinez ${ }^{5}$ and Juan C. Puyana ${ }^{6}$
}

\begin{abstract}
Background: Our objective was to identify possible associations between clinical and laboratory variables and the risk of developing acute kidney injury (AKI) in severely injured patients admitted to the intensive care unit (ICU) for whom creatine kinase (CK) levels were available.

Methods: For this retrospective observational study, we analyzed adult trauma patients admitted to the ICU from 2011 to 2015 at Fundación Valle del Lili (FVL) University Hospital. Our primary outcome was the incidence of AKl. Multivariate regression analysis was used to assess risk factors for this outcome.

Results: A total of 315 patients were included. The trauma mechanisms were blunt $(n=130)$, penetrating $(n=66)$ and blast $(n=44)$ trauma. The median (interquartile range, IQR) of injury severity score (ISS) was $21(16-29)$. AKI developed in 75 patients (23.8\%). Multivariate regression analysis revealed that the thoracic abbreviated injury scale (AIS) value (median (IQR) in the AKI group: 3 (0-4)), Acute Physiology and Chronic Health Evaluation (APACHE II) score (median (IQR) in the AKI group: 18 (10-27)), CK greater than $5000 \mathrm{U} / \mathrm{L}$, lactic acid concentration at admission, and dobutamine administration were independently associated with AKl.

Conclusion: We found that age, APACHE II score, thoracic trauma, lactic acidosis, and dobutamine administration were independently associated with AKI. Trauma surgeons need to be aware of the increased odds of AKI if one of these factors is identified during the evaluation and treatment of injured patients.
\end{abstract}

Keywords: Rhabdomyolysis, Wounds and injuries, Acute kidney injury, Critical care, Trauma

\section{Background}

Organ dysfunction is a common event among trauma patients who survive the initial insult and undergo damage control resuscitation interventions. Acute kidney injury (AKI), which is one type of organ dysfunction, is often present in severely injured patients, and its occurrence is associated with higher risk-adjusted odds of poor outcomes [1], such as greater critical care resource utilization and higher mortality [2].

\footnotetext{
*Correspondence: ramiro.manzano@urosario.edu.co;

ramiro.manzano@fvl.org.co

${ }^{3}$ Escuela de Medicina y Ciencias de la Salud, Universidad del Rosario, Carrera 98 \#18-49, 760001 Bogotá, Colombia

${ }^{4}$ Méderi Hospital Universitario Mayor, Bogotá, Colombia

Full list of author information is available at the end of the article
}

Although previous studies have assessed the frequency and factors associated with AKI in trauma patients, the existing literature about the risk factors for AKI after traumatic injuries has its limitations. Published studies have analyzed patients with traumatic and nontraumatic rhabdomyolysis through univariate analyses [3, 4], and variables that can act as effect modifiers, such as hypoperfusion, transfusions, fluid balance and nephrotoxic substances administration, have not been well investigated. In this study, we analyzed a series of trauma patients admitted to the intensive care unit (ICU) in whom creatine kinase $(\mathrm{CK})$ values were available. Our objective was to identify possible associations between clinical and laboratory variables and the risk of developing AKI in 
severely injured patients admitted to the intensive care unit for whom CK levels were available.

\section{Methods \\ Study design}

A retrospective study was performed to determine the incidence and risk factors for AKI after traumatic rhabdomyolysis.

\section{Data source}

For this study, we retrospectively reviewed the clinical records of patients with trauma from 2011 to 2015 at Fundación Valle del Lili (FVL) University Hospital. FVL is a nonprofit private hospital equivalent to a Level I trauma center affiliated with the ICESI University School of Medicine in Cali-Colombia. It is a 511-bed hospital that serves a referral facility for both civilian and military trauma patients from the southwest region of Colombia. The protocol for this study was approved by the Institutional Review Board (Research Ethics Committee) at FVL.

\section{Patients}

Initial screening identified all trauma patients admitted to FVL from January 2011 to December 2015. We included patients older than 17 years of age who were admitted to the ICU and had CK levels available. Patients with chronic kidney disease, grade IV or V renal injury, known pregnancy, moderate or severe burns, or CK elevation not related to trauma were not eligible. To identify nontraumatic causes of CK elevation, we reviewed patients' past medical history to look for possible causes of nontraumatic CK elevation, such as previously documented rhabdomyolysis, cardiac disease, genetic deficits and use of lipid-lowering drugs.

The primary outcome was the incidence of AKI. There is no consensus on a threshold CK level for diagnosing rhabdomyolysis. However, CK levels are a sensitive indicator of myocyte injury in rhabdomyolysis [5]. Furthermore, elevated CK levels are commonly seen in critically ill patients, and levels of $5000 \mathrm{U} / \mathrm{L}$ or higher are related to the occurrence of renal failure in patients with rhabdomyolysis caused by trauma [6, 7]. Therefore, rhabdomyolysis was defined as a CK level greater than $5000 \mathrm{U} / \mathrm{L}$ in the absence of a recognized etiology such as cardiac disease or muscular dystrophy. Secondary outcomes included the need for renal replacement therapy (RRT) and mortality. AKI was defined according to the KDIGO definitions [8], and patients with any of the three stages according to the KDIGO criteria were analyzed.

Demographics, trauma characteristics, physiological status, resuscitation and therapeutic strategies were reviewed. The Injury Severity Score was calculated according to the AAST grading of injury severity. The
APACHE II score was calculated at day 1 of the ICU admission. RRT was defined as the use of hemodialysis, peritoneal dialysis or continuous RRT. Mortality was defined as death during the hospital stay.

\section{Statistical analysis}

The patients were divided into the AKI group, and the non-AKI group based on final discharge recorded outcomes. The results are summarized with frequencies and percentages for dichotomous and categorical variables and with the mean and standard deviation (SD) or median and interquartile range (IQR) for continuous variables after the analysis of normality with the SK test [9]. Preliminary analysis of the association between potential risk factors and AKI was performed with simple logistic regressions, calculating odds ratios $(O R)$ with their corresponding $95 \%$ confidence intervals $(\mathrm{CI})$ and $P$-values. Variables with a $P<0.1$, as well as clinically relevant variables, were entered into a forward stepwise logistic regression model to identify independent risk factors for the development of AKI, keeping into the model those variables with a $P<0.1$. All analyses were performed with Stata 12.1 (StataCorp, College Station, TX). The goodness of fit of the final model was evaluated with the Hosmer-Lemeshow technique, and the discriminative ability was evaluated with an ROC curve.

\section{Results}

A total of 315 patients were included. Most of the subjects were male $(87.3 \%)$, and three-quarters were younger than 41 years (age, median (IQR): 28.0 (22.0-41.0)). The patients were predominantly victims of blunt trauma $(n=130)$ followed by penetrating trauma $(n=66)$ and blast injuries $(n=44)$. Three-quarters of the patients had an ISS greater than 16 (ISS, median (IQR): 21 (1629)). The median (IQR) of the maximum CK in the first $24 \mathrm{~h}$ was $1662 \mathrm{U} / \mathrm{L}$ (770-4125). A CK greater than 5000 $\mathrm{U} / \mathrm{L}$ was present in 60 patients (19.0\%). AKI developed in 75 patients $(23.8 \%)$. We did not find patients with nontraumatic CK elevation.

Patients were divided into two subgroups: those who developed AKI (AKI group) and those who did not (non-AKI group) (Table 1). Patients were similar in demographic characteristics and admission vital signs. Subjects in the AKI group presented significantly higher abdominal and thoracic Abbreviated Injury Scores (AIS). The proportion of patients with trauma to the extremities as well as the extremity AIS were similar among the patients with and without AKI (60.0\% vs. $64.0 \%$; extremity AIS, median (IQR): $2(0-3)$ vs. $2(0-3)$ ).

Anatomic and physiologic severity scores were significantly higher in patients with AKI than in patients without AKI (ISS, median (IQR): 26 (19-34) vs. 20 (14-27), $P<0.01$; RTS, median (IQR): 7.55 (5.03-7.84) vs. 7.55 
Table 1 Patient characteristics, severity scores and resuscitation strategies

\begin{tabular}{|c|c|c|c|c|}
\hline Variable & Non-AKI group $(n=240)$ & AKI group $(n=75)$ & Total $(n=315)$ & $P$ \\
\hline Male $[n(\%)]$ & $206(85.8)$ & $69(92.0)$ & $275(87.3)$ & 0.17 \\
\hline Age [years, M (IQR)] & $27.0(22.0-38.5)$ & $33.0(22.0-45.0)$ & $28.0(22.0-41.0)$ & 0.15 \\
\hline Trauma mechanism [n(\%)] & & & & 0.01 \\
\hline Penetrating & $66(27.5)$ & $29(38.7)$ & $95(30.2)$ & \\
\hline Blunt & $130(54.2)$ & $42(56.0)$ & $172(54.6)$ & \\
\hline Explosion & $44(18.3)$ & $4(5.3)$ & $48(15.2)$ & \\
\hline $\mathrm{SBP}[\mathrm{mmHg}, \mathrm{M}(\mathrm{IQR})]$ & $119(98-132)$ & $116(95-138)$ & $117(98-133)$ & 0.97 \\
\hline BR [breathings/min, M (IQR)] & $21(18-24)$ & $20(18-26)$ & $21(18-24)$ & 0.62 \\
\hline Glasgow coma score [M (IQR)] & $15(7-15)$ & $14(6-15)$ & $15(7-15)$ & 0.11 \\
\hline AlS head [M (IQR)] & $1(0-4)$ & $0(0-3)$ & $1(0-4)$ & 0.18 \\
\hline AlS face $[M(I Q R)]$ & $0(0-1)$ & $0(0-0)$ & $0(0-1)$ & 0.04 \\
\hline AIS thorax [M (IQR)] & $0(0-3)$ & $3(0-4)$ & $0(0-3)$ & $<0.01$ \\
\hline AIS abdomen/pelvis [M (IQR)] & $0(0-2)$ & $0(0-3)$ & $0(0-2)$ & 0.01 \\
\hline AlS extremity [M (IQR)] & $2(0-3)$ & $2(0-3)$ & $2(0-3)$ & 0.37 \\
\hline AIS external [M (IQR)] & $1(0-1)$ & $1(0-1)$ & $0(0-1)$ & 0.05 \\
\hline ISS [M (IQR)] & $20(14-27)$ & $26(19-34)$ & $21(16-29)$ & $<0.01$ \\
\hline RTS $[M(I Q R)]$ & $7.55(5.97-7.84)$ & $7.55(5.03-7.84)$ & $7.55(5.97-7.84)$ & 0.23 \\
\hline APACHE II [M (IQR)] & $13(8.5-18.5)$ & $18(10-27)$ & $14(9-21)$ & $<0.01$ \\
\hline CK maximun $[\mathrm{M}(\mathrm{IQR})]$ & $1924(726-4940)$ & $2508(999-10,418)$ & $2081(794-5785)$ & 0.02 \\
\hline CK > $5000[n(\%)]$ & $37(15.4)$ & $23(30.7)$ & $60(19.0)$ & $<0.01$ \\
\hline Initial acid lactic [M (IQR)] & $2.98(1.76-4.56)$ & $4.33(2.13-6.09)$ & $3.09(1.77-4.99)$ & $<0.01$ \\
\hline Initial base deficit [M (IQR)] & $7.05(4.5-9.7)$ & $8.7(5.8-12.4)$ & $7.4(4.9-10.2)$ & $<0.01$ \\
\hline Fluid balance, first $24 \mathrm{~h}$ [Lt., M (IQR)] & $1.96(0.64-3.42)$ & $3.37(1.57-5.49)$ & $2.19(0.83-3.81)$ & $<0.01$ \\
\hline Fluid balance $72 \mathrm{~h}$ [Lt., M (IQR)] & $2.83(0.27-5.18)$ & $6.28(3.05-9.48)$ & $3.33(0.89-6.42)$ & $<0.01$ \\
\hline Packed red blood cells, first $24 \mathrm{~h}[\mathrm{U}, \mathrm{M}(\mathrm{IQR})]$ & $0(0-3)$ & $2(0-4)$ & $0(0-4)$ & $<0.01$ \\
\hline Number of patients requiring FFP transfusion $[n(\%)]$ & $52(21.7)$ & $29(38.7)$ & $23(7.3 \%)$ & $<0.01$ \\
\hline Administration of $\mathrm{NaH}_{2} \mathrm{CO}_{3}[n(\%)]$ & $16(6.7)$ & $11(14.7)$ & $27(8.6)$ & 0.03 \\
\hline Contrast administration $[n(\%)]$ & $149(62.1)$ & $52(69.3)$ & $201(63.8)$ & 0.25 \\
\hline Contrast dose $\times 100 \mathrm{mg}[\mathrm{M}(\mathrm{IQR})]$ & $1.0(0-1.5)$ & $1.0(0-2.0)$ & $1(0-2)$ & 0.15 \\
\hline Norephinephrine administration [n(\%)] & $92(38.3)$ & $45(60.0)$ & $173(54.9)$ & $<0.01$ \\
\hline Vasopresina administration $[n(\%)]$ & $30(12.5)$ & $14(18.7)$ & $44(14.0)$ & 0.18 \\
\hline Dobutamine administration [n(\%)] & $9(3.7)$ & $11(14.7)$ & $20(6.3)$ & $<0.01$ \\
\hline
\end{tabular}

SBP Systolic blood pressure, BR Breathing rate, AIS Abbreviate injury score, ISS Injury severity score, $R T S$ Revised trauma score, $\mathrm{CK}_{\mathrm{Creatine}}$ kinase, $\mathrm{NaH}_{2} \mathrm{CO}_{3}$ Sodium bicarbonate

(5.97-7.84), $P=0.23$ APACHE II, median (IQR): 18 (10$27)$ vs. 13 (8.5-18.5); $P<0.01)$.

Individuals in the AKI group were more likely to have significantly higher base deficit and lactate values $(P<0.01)$. The proportion of patients with a CK greater than $5000 \mathrm{U} / \mathrm{L}$ was significantly higher in the AKI group (23 of 75 of patients with AKI (30.7\%) vs. 37 of 240 of patients without AKI (15.4\%); $P<0.01)$.

Among the therapeutic interventions within the first $24 \mathrm{~h}$ after admission, a significantly higher number of patients in the AKI group required vasoactive drugs. Norepinephrine was used in 60.0 and $38.3 \%$ of patients with and without AKI, respectively $(P<0.01)$. Dobutamine was required in 14.7 and $6.3 \%$ of patients with and without AKI, respectively $(P<0.01)$.

In the first $24 \mathrm{~h}$ after admission, significantly more patients in the AKI group than in the group of patients without AKI received plasma transfusions (29 of 75 patients with AKI (38.7\%) vs. 52 of 240 patients without AKI (21.7\%)). Furthermore, patients with AKI received significantly higher volumes of crystalloids in the first $72 \mathrm{~h}$ and packed red blood cells transfusions in the first $24 \mathrm{~h}$ and were more likely to receive sodium bicarbonate than patients without AKI (Table 1). 
There were significant differences in mortality between AKI-KDIGO groups $(P<0.001$, Fig. 1$)$. The overall mortality was $13 \%$. However, the highest mortality occurred in the patients with KDIGO stage 3 AKI (Fig. 1).

In the univariate analysis, a CK greater than $5000 \mathrm{U} / \mathrm{L}$ showed a positive association with AKI risk $(O R=2.35$, 95\%CI 1.29-4.31). After multivariate regression analysis, we found that age, thoracic AIS value (median (IQR) in the AKI group: 3 (0-4)), APACHE II score (median (IQR) in the AKI group: 18 (10-27)), CK greater than $5000 \mathrm{U} / \mathrm{L}$, lactic acid concentration at admission, and dobutamine administration were independently associated with AKI in our study population (Table 2). The final regression model demonstrated adequate calibration $(P=0.25)$ and acceptable discrimination ((area under curve $($ AUC $)=0.767$, 95\%CI 0.703-0.832).

\section{Discussion}

This study investigated the risk factors of AKI in trauma patients admitted to the ICU for whom CK results were available. We showed that patients with AKI were more likely to suffer from perfusion derangement, had worse injuries and required more aggressive resuscitation strategies. When adjusting for confounders, we found that increased age, dobutamine administration, the severity of the thoracic injury, the severity of disease (APACHE II), the initial lactic acid level and a CK value greater than $5000 \mathrm{U} / \mathrm{L}$ were associated with the occurrence of AKI. Our findings are similar to those reported in a larger multicenter study [10], in which the severity of the trauma, the value of lactate and the presence of hemorrhagic shock were independently associated with the development of AKI. Thus, it is rational to posit that harsh physiological exhaustion driven by the severity of trauma may play a key role in the development of AKI among trauma patients.

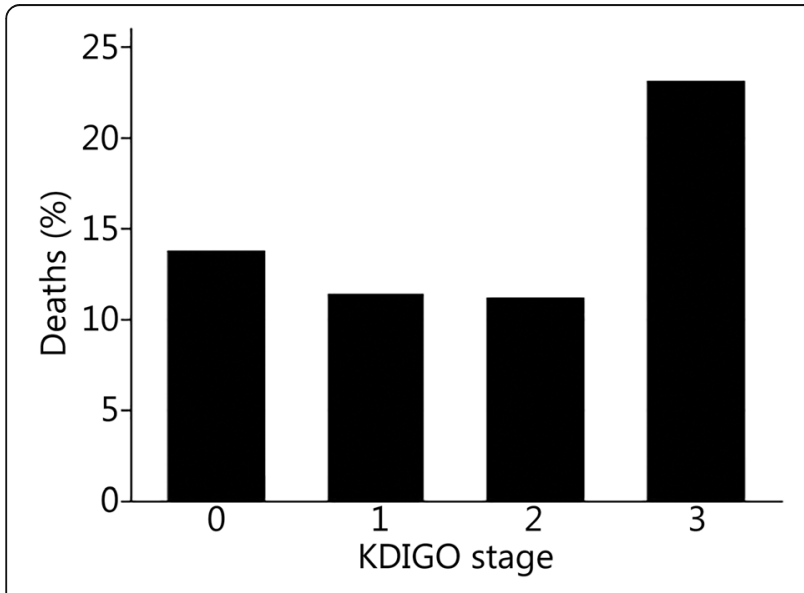

Fig. 1 Mortality by acute kidney injury KDIGO stage
We found that increasing age and a CK value greater than $5000 \mathrm{U} / \mathrm{L}$ were independently associated with AKI. Age has been uniformly identified in the analysis of risk factors of posttraumatic AKI or multiorgan failure [1114]. Regarding the role of CK, our results seem to be consistent with previous research, which found that abnormal CK levels are related to renal failure in patients with rhabdomyolysis caused by trauma $[3,6,7]$. For example, Brown et al. [7] analyzed data from 2083 trauma admissions to the intensive care unit and found that a CK value greater than $5000 \mathrm{U} / \mathrm{L}$ was independently associated with renal failure. Similarly, Sharp et al. [3] found that an elevated serum CK value was a significant risk factor for AKI. Studies performed in war fighters have confirmed this association and have reported a positive correlation between AKI stages and higher CK values $[4,15]$.

Once a trauma patient is diagnosed with rhabdomyolysis, his or her prognosis depends heavily on a timely implemented and adequate therapeutic strategy. In addition to rehydration with IV fluids, other interventions such as mannitol and bicarbonate administration have been proposed to prevent AKI $[6,16]$. However, the use of bicarbonate to alkalinize the urine remains controversial [17], as no randomized clinical trial supports its use. In this sense, our univariate analysis showed that the administration of sodium bicarbonate was associated with AKI. However, this association could be biased because the administration of bicarbonate could be used for treating metabolic acidosis not to prevent AKI or for a random treatment; our results are consistent with those of Brown et al. [7], who showed that therapy with bicarbonate and mannitol administration was independently associated with renal failure. Furthermore, the administration of bicarbonate in critically ill trauma and nontrauma patients with metabolic acidosis has been shown to paradoxically increase the $\mathrm{CO}_{2}$ arterial pressure and thus cellular metabolic acidosis $[18,19]$. In contrast, Nielsen et al. [20] showed better outcomes among subjects with traumatic rhabdomyolysis treated with a protocol of forced alkaline diuresis. Beyond bicarbonate administration, it is well known that early IV fluid administration is paramount for improving outcomes among patients with elevated CK levels at risk of AKI [21]; nevertheless, overaggressive fluid therapy has deleterious effects in trauma patients [22]. Although resuscitation strategies with fluid infusion rates of $1 \mathrm{~L} / \mathrm{h}$ for $2 \mathrm{~h}$ after injury and $500 \mathrm{ml} /$ $\mathrm{h}$ after $120 \mathrm{~min}[21,23]$ are found in the literature, these recommendations are not supported by randomized controlled trials; thus, we advise caution when infusing large amounts of fluids in patients with AKI secondary to rhabdomyolysis due to trauma.

The current study found that lactic acid elevation and dobutamine administration were independently associated with the development of AKI. Previous studies have revealed an association between impeding hypoperfusion 
Table 2 Risk factors associated with acute kidney dysfunction in traumatic rhabdomyolysis

\begin{tabular}{|c|c|c|c|c|}
\hline \multirow[t]{2}{*}{ Variables } & \multicolumn{2}{|c|}{ Simple logistic regressions } & \multicolumn{2}{|c|}{ Multivariable logistic regression $^{a}$} \\
\hline & $O R(95 \% \mathrm{Cl})$ & $P$ & OR $(95 \% \mathrm{Cl})$ & $P$ \\
\hline \multicolumn{5}{|l|}{ Demographics } \\
\hline Age (for every 10 years) & $1.16(0.98-1.38)$ & 0.083 & $1.30(1.06-1.60)$ & 0.01 \\
\hline \multicolumn{5}{|l|}{ Gender } \\
\hline Male & $0.53(0.2-1.31)$ & 0.167 & - & \\
\hline Female & - & & & \\
\hline \multicolumn{5}{|l|}{ Trauma mechanism } \\
\hline Blast & - & & $0.334(0.10-1.12)$ & 0.08 \\
\hline Penetrating & $3.56(1.21-10.48)$ & 0.022 & & \\
\hline Blunt & $4.83(1.59-14.71)$ & 0.006 & & \\
\hline \multicolumn{5}{|l|}{ Severity scores } \\
\hline RTS & $0.82(0.69-0.98)$ & 0.026 & - & \\
\hline ISS & $1.04(1.01-1.06)$ & 0.003 & - & \\
\hline AIS head \& neck & $0.89(0.78-1.03)$ & 0.118 & - & \\
\hline AIS face & $0.78(0.59-1.02)$ & 0.068 & - & \\
\hline AIS thorax & $1.30(1.12-1.51)$ & $<0.001$ & $1.23(1.04-1.46)$ & 0.02 \\
\hline AIS abdomen/pelvis & $1.23(1.06-1.43)$ & 0.006 & - & \\
\hline AIS extremity & $1.09(0.93-1.29)$ & 0.293 & - & \\
\hline AIS external & $0.71(0.50-1.00)$ & 0.052 & - & \\
\hline APACHE ॥ & $1.08(1.05-1.12)$ & $<0.01$ & $1.05(1.01-1.09)$ & 0.03 \\
\hline \multicolumn{5}{|l|}{ Biochemical markers } \\
\hline Initial lactic acid & $1.23(1.11-1.36)$ & $<0.001$ & $1.17(1.03-1.33)$ & 0.02 \\
\hline Initial base deficit & $1.12(1.06-1.19)$ & $<0.01$ & - & \\
\hline Higher CK & $1.00(0.99-1.0)$ & 0.12 & - & \\
\hline $\mathrm{CK}>5000 \mathrm{U} / \mathrm{L}$ & $2.35(1.29-4.31)$ & $<0.01$ & $2.64(1.31-5.33)$ & $<0.001$ \\
\hline \multicolumn{5}{|l|}{ Resuscitation \& nephrotoxic agents } \\
\hline Fluid balance, first day & $1.16(1.06-1.27)$ & $<0.01$ & - & \\
\hline Fluid balance $72 \mathrm{~h}$ & $1.20(1.12-1.28)$ & $<0.01$ & - & \\
\hline Packed red blood cells, first day & $1.09(1.02-1.17)$ & 0.011 & - & \\
\hline FFP transfusion & $2.28(1.31-3.98)$ & $<0.01$ & - & \\
\hline Bicarbonate administration & $2.4(1.96-5.44)$ & 0.035 & - & \\
\hline Contrast administration & $1.43(0.80-2.54)$ & 0.23 & - & \\
\hline Contrast dose $\times 100 \mathrm{mg}$ & $1.32(1.04-1.67)$ & 0.02 & - & \\
\hline Norepinephrine administration & $2.42(1.42-4.1)$ & $<0.01$ & - & \\
\hline Vasopressin administration & $1.59(0.79-3.19)$ & 0.19 & - & \\
\hline Dobutamine administration & $4.37(1.74-11.0)$ & $<0.01$ & $3.16(1.01-9.94)$ & 0.049 \\
\hline
\end{tabular}

${ }^{a}$ Stepwise multivariable logistic regression model with backwards elimination. Variables with $P$ value $<0.1$ were retained in the model. OR Odds ratio, $C I$ Confidence interval, RTS Revised trauma score, ISS Injury severity score, AIS Abbreviate injury scale, CK Creatine kinase, FFP Fresh frozen plasma

(increased lactate and base deficit) and a greater odds of AKI in trauma patients [3, 24]. For example, Bihorac et al. [24] found that severe trauma patients with a lactate level of $5 \mathrm{mmol} / \mathrm{L}$ in the first $24 \mathrm{~h}$ had a $25 \%$ chance of developing AKI, and this probability increased proportionally with lactate levels. As lactic acid has been proposed as an indicator of resuscitation adequacy [25-27], these results could also mean that acidotic patients who required dobutamine administration were inadequately resuscitated during the early trauma care or had a poor response to volume resuscitation [28]. Regarding the latter, Morris et al. [29] reported that approximately one-third of patients developed early posttraumatic AKI resulted from inappropriate resuscitation. Furthermore, it has been 
reported that hypotension adversely influences the prognosis of AKI in trauma patients [30].

The association between thoracic trauma and AKI that we found has not been previously reported. Although it is not clear how thoracic injuries could increase the odds of AKI after traumatic rhabdomyolysis, there are possible explanations. Thoracic trauma causes a disturbed immunologic response characterized by elevations in proinflammatory cytokines that can result in multiorgan failure $[14,31,32]$. Therefore, it is plausible to posit that severe thoracic injuries induce persistent posttraumatic inflammation, which in turn results in worse outcomes, such as AKI and multiorgan failure.

In this study, mortality was significantly higher in the group of patients who had KDIGO-3 AKI (Fig. 1). This result suggests a biologic gradient between worse renal function and mortality [24,33] and that the chances of death are proportional to the severity of AKI. Although we did not analyze the predictors of mortality in our series, it is important to mention the study by Stewart et al. [15]. They examined a large cohort of combat casualties to identify risk factors for rhabdomyolysis and its relation to AKI and death. Beyond showing that patients with AKI had a higher mortality risk, they demonstrated that rhabdomyolysis acted as an effect modifier in the pathway between AKI and death.

\section{Limitations}

Our study is not without limitations, and the results should be interpreted in the context of the study design. First, the retrospective nature of the investigation makes it prone to bias and confusion. Second, further selection bias could be introduced as only patients in whom a CK test was performed were included. Third, the etiology of AKI is usually multifactorial, and rhabdomyolysis may explain the development of AKI only in a proportion of critically ill patients. Furthermore, the fact that our patients were those who underwent CK testing could compromise the external validity. Because we included only patients who had a CK test, our results could be pervaded by selection bias. Therefore, our results can only be extrapolated to a small population in which the pretest probability of rhabdomyolysis and AKI is higher than that in other populations. In other words, our results can be extrapolated to those patients in whom clinical awareness of traumatic rhabdomyolysis is greater. Furthermore, despite our regression analysis, there could be persistent differences in some of the variables (i.e., trauma mechanism) among the groups compared, suggesting that residual confounding affected our analysis.

Finally, a key strength of our analysis is the construction of a multivariable model that included known susceptibility and risk factors for AKI such as age, trauma mechanisms, hypoperfusion variables, resuscitation variables and toxic or protector substance administration.

\section{Conclusion}

We found that age, APACHE II score, thoracic trauma, lactic acidosis, and dobutamine administration were independently associated with AKI. Trauma surgeons need to be aware of the increased odds of AKI if one of these factors is identified during the evaluation and treatment of injured patients.

\section{Abbreviations \\ AIS: Abbreviated injury scale; AKI: Acute kidney injury; APACHE II: Acute physiology and chronic health evaluation; AUC: Area under curve; $\mathrm{Cl}$ : Confidence intervals; CK: Creatine kinase; ICU: Intensive care unit; IQR: Interquartile range; ISS: Injury severity score; OR: Odds ratios}

\section{Acknowledgements}

Presented at the Clinical Congress of the American College of Surgeons, October 16-20, 2017 in Washington, MD.

\section{Authors' contributions}

Only individuals who made significant contributions were selected as authors. AFG, RMN, JGB, MPN, DNV, MM, SO, JMM, NM and JCP contributed equally to this work. AFG conceived the research question. JGB, MPN DNV, MM, SO, JMM and NM collected the data and performed a literature search. AFG and JCP performed the statistical analysis. RMN and AFG wrote the first draft of the manuscript. All authors revised the first draft of the manuscript and made substantial contributions to the final version for submission. The authors read and approved the final manuscript.

\section{Funding}

This work was supported in part by the National Institutes of Health John E. Fogarty International Center grant\#D43 TW007560. The funding organization had no role in the design and conduct of the study, in the collection, management, analysis, and interpretation of the data, or in the preparation, review, or approval of the manuscript.

Availability of data and materials

Data are the property of the authors and can be obtained by contacting the Principal Investigator: Dr. Alberto F. García; e-mail: alberto.garcia@correounivalle.edu.co

Ethics approval and consent to participate

The protocol for this study was approved by the Fundación Valle del Lili Institutional Review Board in Cali, Colombia. Consent to participate was not necessary because the study was retrospective.

\section{Consent for publication}

Not applicable.

\section{Competing interests}

The authors declare that they have no competing interests.

\section{Author details}

${ }^{1}$ Division of Trauma and Acute Care Surgery, Department of Surgery, Fundación Valle del Lili, Cali, Colombia. ${ }^{2}$ Clinical Research Center, Fundación Valle del Lili, Cali, Colombia. 'Escuela de Medicina y Ciencias de la Salud, Universidad del Rosario, Carrera 98 \#18-49, 760001 Bogotá, Colombia. ${ }^{4}$ Méderi Hospital Universitario Mayor, Bogotá, Colombia. ${ }^{5}$ School of Medicine, Universidad ICESI (ICESI University), Cali, Colombia. ${ }^{6}$ Department of Surgery, University of Pittsburgh, Pittsburgh, PA, USA. 
Received: 10 February 2020 Accepted: 28 September 2020

Published online: 09 October 2020

\section{References}

1. Harrois A, Libert N, Duranteau J. Acute kidney injury in trauma patients. Curr Opin Crit Care. 2017;23(6):447-56

2. Søvik S, Isachsen MS, Nordhuus KM, Tveiten CK, Eken T, Sunde K, et al. Acute kidney injury in trauma patients admitted to the ICU: a systematic review and meta-analysis. Intensive Care Med. 2019:45(4):407-19.

3. Sharp LS, Rozycki GS, Feliciano DV. Rhabdomyolysis and secondary renal failure in critically ill surgical patients. Am J Surg. 2004;188(6):801-6.

4. Elterman J, Zonies D, Stewart I, Fang R, Schreiber M. Rhabdomyolysis and acute kidney injury in the injured war fighter. J Trauma Acute Care Surg. 2015;79(4 Suppl 2):S171-4.

5. El-Abdellati E, Eyselbergs M, Sirimsi H, Van Hoof V, Wouters K, Verbrugghe W, et al. An observational study on rhabdomyolysis in the intensive care unit. Exploring its risk factors and main complication: Acute kidney injury. Ann Intensive Care. 2013;3(1):8

6. Huerta-Alardín AL, Varon J, Marik PE. Bench-to-bedside review: Rhabdomyolysis - an overview for clinicians. Crit Care. 2005;9(2):158-69.

7. Brown C, Rhee P, Chan L, Evans K, Demetriades D, Velmahos G. Preventing renal failure in patients with rhabdomyolysis: do bicarbonate and mannito make a difference? J Trauma Acute Care Surg. 2004;56(6):1191-6.

8. Kellum JA, Lameire N. Diagnosis, evaluation, and management of acute kidney injury: a KDIGO summary (part 1). Crit Care. 2013;17(1):204.

9. D'Agostino RB, Belanger A. A suggestion for using powerful and informative tests of normality. Am Stat. 1990:44(4):316-21.

10. Harrois A, Soyer B, Gauss T, Hamada S, Raux M, Duranteau J, et al. Prevalence and risk factors for acute kidney injury among trauma patients: a multicenter cohort study. Crit Care. 2018;22(1):344.

11. Sauaia A, Moore FA, Moore EE, Haenel JB, Read RA, Lezotte DC. Early predictors of postinjury multiple organ failure. Arch Surg. 1994;129(1):39-45.

12. Vogel JA, Liao MM, Hopkins E, Seleno N, Byyny RL, Moore EE, et al. Prediction of postinjury multiple-organ failure in the emergency department: development of the Denver emergency department trauma organ failure score. J Trauma Acute Care Surg. 2014;76(1):140-5.

13. Dewar DC, Tarrant SM, King KL, Balogh ZJ. Changes in the epidemiology and prediction of multiple-organ failure after injury. J Trauma Acute Care Surg. 2013;74(3):774-9.

14. Fröhlich $M$, Lefering $R$, Probst $C$, Paffrath $T$, Schneider MM, Maegele $M$, et al. Epidemiology and risk factors of multiple-organ failure after multiple trauma: an analysis of 31,154 patients from the TraumaRegister DGU. Trauma Acute Care Surg. 2014;76(4):921-7.

15. Stewart IJ, Faulk TI, Sosnov JA, Clemens MS, Elterman J, Ross JD, et al. Rhabdomyolysis among critically ill combat casualties: associations with acute kidney injury and mortality. J Trauma Acute Care Surg. 2016;80(3): 492-8.

16. Bosch X, Poch E, Grau JM. Rhabdomyolysis and acute kidney injury. N Engl J Med. 2009:361(1):62-72.

17. Scharman EJ, Troutman WG. Prevention of kidney injury following rhabdomyolysis: a systematic review. Ann Pharmacother. 2013:47(1):90-105.

18. Wilson RF, Spencer AR, Tyburski JG, Dolman H, Zimmerman LH. Bicarbonate therapy in severely acidotic trauma patients increases mortality. J Trauma Acute Care Surg. 2013;74(1):45-50.

19. Cooper D, Walley K, Wiggs B, Russell J. Bicarbonate does not improve hemodynamics in critically ill patients who have lactic acidosis: a prospective, controlled clinical study. Ann Intern Med. 1990;112(7):492-8.

20. Nielsen JS, Sally M, Mullins RJ, Slater M, Groat T, Gao X, et al. Bicarbonate and mannitol treatment for traumatic rhabdomyolysis revisited. Am J Surg. 2017;213(1):73-9.

21. Chavez LO, Leon M, Einav S, Varon J. Beyond muscle destruction: a systematic review of rhabdomyolysis for clinical practice. Crit Care. 2016; 20(1):135.

22. Cotton BA, Guy JS, Morris JAJ, Abumrad NN. The cellular, metabolic, and systemic consequences of aggressive fluid resuscitation strategies. Shock. 2006;26(2):115-21.

23. Sever MS, Vanholder R, Ashkenazi I, Becker G, Better O, Covic A, et al. Recommendations for the management of crush victims in mass disasters. Nephrol Dial Transplant. 2012;27(Suppl_1):i1-67.
24. Bihorac A, Delano MJ, Schold JD, Lopez MC, Nathens AB, Maier RV, et al Incidence, clinical predictors, genomics, and outcome of acute kidney injury among trauma patients. Ann Surg. 2010;252(1):158-65.

25. Odom SR, Howell MD, Silva GS, Nielsen VM, Gupta A, Shapiro NI, et al, Lactate clearance as a predictor of mortality in trauma patients. J Trauma Acute Care Surg. 2013;74(4):999-1004.

26. Régnier M-A, Raux M, Le Manach Y, Asencio Y, Gaillard J, Devilliers C, et al. Prognostic significance of blood lactate and lactate clearance in trauma patients. Anesthesiology. 2012;117(6):1276-88.

27. Dezman ZD, Comer AC, Smith GS, Narayan M, Scalea TM, Hirshon JM. Failure to clear elevated lactate predicts 24-hour mortality in trauma patients. J Trauma Acute Care Surg. 2015;79(4):580-5.

28. Miller PR, Meredith JW, Chang MC. Randomized, prospective comparison of increased preload versus inotropes in the resuscitation of trauma patients: effects on cardiopulmonary function and visceral perfusion. J Trauma Acute Care Surg. 1998:44(1):107-13

29. Morris JA, Mucha P, Ross SE, Moore BF, Hoyt DB, Gentilello L, et al. Acute posttraumatic renal failure: A multicenter perspective. J Trauma. 1991;31(12): 1584-90.

30. Nadvi SS, Mokoena T, Gouws E, Haffejee AA. Prognosis in posttraumatic acute renal failure is adversely influenced by hypotension and hyperkalaemia. Eur J Surg. 1996:162(2):121-4.

31. Roumen RM, Hendriks T, van der Ven-Jongekrijg J, Nieuwenhuijzen GA, Sauerwein RW, van der Meer JW, et al. Cytokine patterns in patients after major vascular surgery, hemorrhagic shock, and severe blunt trauma. Relation with subsequent adult respiratory distress syndrome and multiple organ failure. Ann Surg. 1993;218(6):769-76

32. Jastrow KM, Gonzalez EA, McGuire MF, Suliburk JW, Kozar RA, lyengar S, et al. Early cytokine production risk stratifies trauma patients for multiple organ failure. J Am Coll Surg. 2009;209(3):320-31.

33. Bagshaw SM, George C, Gibney RTN, Bellomo R. A multi-center evaluation of early acute kidney injury in critically ill trauma patients. Ren Fail. 2008; 30(6):581-9.

Ready to submit your research? Choose BMC and benefit from

- fast, convenient online submission

- thorough peer review by experienced researchers in your field

- rapid publication on acceptance

- support for research data, including large and complex data types

- gold Open Access which fosters wider collaboration and increased citations

- maximum visibility for your research: over $100 \mathrm{M}$ website views per year

At $\mathrm{BMC}$, research is always in progress.

Learn more biomedcentral.com/submissions 